\title{
Approaches to functional genomics in filamentous fungi
}

\author{
Richard J Weld ${ }^{1}$, Kim M Plummer ${ }^{1,2}$, Margaret A Carpenter ${ }^{1}$, Hayley J Ridgway ${ }^{1}$ \\ ${ }^{I}$ National Centre for Advanced Bio-Protection Technologies, PO Box 84, Lincoln University, Canterbury 8150, New Zealand; ${ }^{2}$ LaTrobe \\ University, Melbourne 3086, Australia
}

The study of gene function in filamentous fungi is a field of research that has made great advances in very recent years. A number of transformation and gene manipulation strategies have been developed and applied to a diverse and rapidly expanding list of economically important filamentous fungi and oomycetes. With the significant number of fungal genomes now sequenced or being sequenced, functional genomics promises to uncover a great deal of new information in coming years. This review discusses recent advances that have been made in examining gene function in filamentous fungi and describes the advantages and limitations of the different approaches.

Cell Research (2006) 16:31-44. doi:10.1038/sj.cr.7310006; published online 16 January 2006

Keywords: functional genomics, gene replacement, filamentous fungi, homologous recombination, RNAi, gene silencing, oomycetes

\section{Introduction}

In recent years there has been a surge of interest in functional genomics research in filamentous fungi and oomycetes. This has been facilitated by several important advances. Firstly, the development of various transformation systems has steadily increased the number of filamentous fungi, and the variety of fungal tissue types, that are amenable to genetic manipulation. Secondly, techniques for random mutagenesis and targeted gene mutation have been adapted and developed as efficient tools for investigating gene function in fungi. Thirdly, the genomes of a significant number of fungi have been, or are presently being, sequenced (http://www.genomesonline. org/). Despite the multiple tools now available, the initiation of a new functional genomics project is not always straightforward.

There are many possible combinations of transformation systems and functional genomics strategies available, not all of which are standard practice in filamentous fungi. Tailoring the right strategy for a particular project is a complicated task with many variables to consider. While

Correspondence: Richard J Weld

Tel: +64-03-3253696; Fax: +64-03-3253864;

E-mail: weldr@lincoln.ac.nz
Agrobacterium-mediated transformation (AMT) has been developed as a powerful tool for both random and targeted gene disruption and is increasingly being seen as the system of choice for many fungi (reviewed in [1]), there are other methods that offer advantages in specific situations. Also, some successful transformation systems may not be suitable for particular uses. Frequency of homologous recombination, for example, is highly dependent on the mode of transformation [2]. In this review we provide an overview of the different approaches to transformation and manipulation of gene expression in filamentous fungi and describe strategies that have been used successfully for fungal functional genomics.

There are a number of reviews on transformation and functional genomics of fungi in the literature. Some of these reviews are restricted to quite specific areas of this topic. AMT and its application to functional genomics in fungi has been comprehensively reviewed recently [1]. A review of RNA silencing in fungi, still in press at the time of writing, by Hitoshi Nakayashiki has just been completed [3]. While our review covers some of the material in those papers, we have sought to give a broader, comparative overview of the approaches to functional genomics in filamentous fungi and to indicate the directions in which this research is heading. Other general reviews are also available $[4,5]$ and this review updates that work with recent advances. 


\section{Achieving stable, homokaryotic transformation}

Most functional genomics methods depend upon the production of stable transformants. In recent years, efficient transformation systems have been developed for a wide range of filamentous fungi (reviewed in $[1,4,5]$ ). Successful transformation of fungi has been achieved by: $\mathrm{CaCl}_{2}$ /polyethylene glycol [6-8], electroporation [9-15], particle bombardment [2, 6, 10, 16, 17], and Agrobacterium tumefaciens-mediated transformation (AMT) [1].

Transformation of protoplasts using $\mathrm{CaCl}_{2}$ /polyethylene glycol (PEG) typically occurs in 15-30 min incubation at room temperature in the presence of $10-50 \mathrm{mM} \mathrm{CaCl}_{2}$ and high concentrations of PEG. Electroporation is also usually used to transform protoplasts, though sometimes germinating conidia are used. During electroporation, protoplasts or conidia are exposed briefly to a high amplitude electric field, which permeabilizes the membrane permitting uptake of foreign DNA. Although used widely, a major limitation of these methods is that they usually require the production of protoplasts, a process that suffers from inconsistencies between the enzyme batches used to digest fungal cell walls and the ability of cell walls from different fungi to be digested $[16,18]$.

For particle bombardment (biolistics), gold or tungsten beads are coated with the transforming DNA and used to bombard the intact fungal tissue [2, 18-21]. The flexibility of target tissues used for biolistics has advantages in strains that produce little material suitable for protoplast production $[18,22]$. Biolistics is also useful in systems where protoplasting has not been developed and there is little known about the components of the cell wall [23, 24]. Many researchers have reported similar or increased frequency of transformation, complexity of integration events and mitotic stability compared with protoplast mediated transformation [2, 18-21,25], although direct comparisons are difficult due to different target tissues, fungal genera and optimisation protocols.

A recent trend has been towards functional genomics approaches based on AMT. AMT has been shown to produce a significantly higher frequency of transformation and more stable transformants with less complicated insertional mutants than biolistics $[26,27]$. Under the right conditions, A. tumefaciens is able to transfer DNA (T-DNA) to a very wide range of fungi and fungal tissues. Fungi that have been recalcitrant to transformation by other systems have been successfully transformed by co-cultivation with Agrobacterium [27]. AMT is a relatively simple system to work with, does not require the production of protoplasts, and is suitable for both gene-replacement by homologous recombination $[1,28,29]$ and insertional mutagenesis by random integration [30, 31].
Despite these advances, the development of a new transformation system is often not as straightforward as the literature suggests, which is biased towards success. Even for AMT there are so many variables that need to be optimised for individual systems that the selection of a particular combination of parameters that work may often be more fortuitous than systematic. Once a transformation system has been successfully developed, there may still be major barriers to overcome before it is possible to analyse gene function. A potential problem for genetic analysis is the multinucleate nature of filamentous fungi. The presence of multiple nuclei can confound methods, such as gene replacement and insertional mutagenesis, which rely on the isolation of homokaryotic transformants derived from a single transformation event to study loss of function mutants [32].

In transformed multinucleate yeast, where the cells are heterokaryotic for the transformation event, homokaryotic tissue is produced within a single round of colony growth, probably by random assortment of nuclei during budding [33]. By growing colonies on selective media that kills cells that are homokaryotic for untransformed nuclei, transformed, homokaryotic tissue is easily isolated. A similar effect may work in filamentous fungi as it has long been known that the proportion of genetically different nuclei within hyphae is responsive to selective pressure [34]. In filamentous fungi, selection can also produce homokaryotic tissue from transformed tissue that must originally have been heterokaryotic [35], though this may not always happen $[18,36]$. Where gene disruption results in a loss of function that impairs growth, selection for transformants is likely to produce heterokaryotic tissue as tissue that is homokaryotic for either mutant or wild-type nuclei will suffer a selective disadvantage [29,37]. This problem might be overcome if it is possible to transform uninucleate tissue or to cycle transformed tissue through a uninucleate stage $[18,29,38]$.

Difficulties producing homokaryotic transformants from multinucleate tissue can be avoided by using techniques that are not compromised by the presence of the wild-type gene. If the target gene sequence is known, it is possible to use gene expression knockdown strategies, such as RNAi, that rely on inactivation of mRNA rather than gene mutation $[39,40]$. However, for random mutagenesis strategies which seek to find unknown genes through phenotypic changes, the presence of untransformed nuclei or nuclei with different integration loci, may prove more challenging.

\section{Marker genes for transformation}

A wide range of genes have been found to be suitable as selectable markers for fungi. The $h p h$ gene (hygromycin 
B resistance) is the most commonly used selection system because of its effectiveness in most, but not all, systems. Other selective agents such as phleomycin, sulfonylurea, nourseothricin, bialophos, carboxin, blasticidin $\mathrm{S}$ and benomyl have also been used [5, 41-44]. An alternative to drug resistance genes for transformation of fungi is to use auxotrophic markers such as $\operatorname{pyr} G$ (a homologue of the Saccharomyces cerevisiae ura3 gene). Mutants that lack pyr $G$ are auxotrophic for uracil so vectors containing $p y r G$ allow selection on uracil-deficient media [45]. Additionally, pyrG-deficient mutants are resistant to 5-fluoro-orotic acid (5FOA) which is toxic in prototrophs. Positive/negative selection genes such as $p y r G$ provide the possibility of conducting a series of sequential transformation by using Blaster cassettes [46].

Blaster cassettes, based on URA3, have allowed sequential transformation in yeast and have now been developed for use in filamentous fungi [47]. In the blaster cassette, the $u \mathrm{ra3} / \mathrm{pyr} G$ gene is flanked by two small direct repeat sequences. Carried on the transforming DNA, the blaster cassette allows selection for transformants on medium lacking uracil. Once transformants are isolated, the ura3/ pyr $G$ gene can be excised by recombination between the two direct repeat sequences, returning the transformants to uracil prototrophy. Transformants that have excised the ura3/pyrG gene are selected in the presence of 5FOA and can be re-transformed with another vector carrying the blaster cassette.

It is important to note that there is a problem using URA3 as a marker for gene disruption. The virulence phenotype of Candida albicans has been shown to vary dependent upon the position of the URA3 marker gene [48]. The activity of the enzyme encoded by URA3 is to some extent loci dependent and has an influence on virulence. This phenomenon is a problem for studies using URA3 in studies seeking to correlate gene knockout with virulence.

\section{Random insertional mutagenesis (gene tagging)}

By inserting DNA into the genome, either through transformation or by the in vivo movement of mobile genetic elements, a genome-wide series of random, tagged mutations can be created. Depending on the composition of the genetic element, it is possible to disrupt genes, to tag promoters or enhancers, or to up-regulate genes. Transformed isolates are selected and examined for phenotypic changes of interest. On the assumption that the phenotype change is due to genetic disruption wrought by the transferred DNA, the genomic region contiguous with the inserted T-DNA is retrieved either by PCR-based methods such as inverse PCR and TAIL-PCR or by plasmid rescue [31]. If there are many tandem integrations, semi-random PCR can be used to recover genomic DNA [49].

Ideally, a gene tagging system should have: a high transformation frequency; random integration as a single copy, at a single locus without rearrangement or deletion of either the transferred DNA or the genomic DNA; and, since all transformation methods induce unexpected genomic lesions, a straightforward method, such as sexual crosses, to test the correlation of the mutant phenotype with the presence of the DNA tag. If genetic segregation cannot be used, another strategy to rapidly check that the integration site correlates with the phenotype is to use plasmid rescue to create a gene disruption vector. The rescued plasmid can then be used to disrupt the same locus in a wild-type strain and do a complementation test [50].

\section{Gene tagging by direct DNA transfer}

Direct DNA transfer has been, and is still being, used successfully as a method for random mutagenesis in fungi [50-53]. As DNA that is not homologous to the recipient genome appears to integrate approximately at random [54], thousands of individual transformants collectively provide a library of tagged mutations. This method has worked successfully for the discovery of new genes correlated to a particular mutant phenotype. However, the generation of thousands of mutants by generally low efficiency transformation methods is not always feasible. Also, plasmid integration may result in a high frequency of tandem-repeat integrations. Tandem integrations probably occur through an initial integration at a chromosomal break or nick, followed by homologous recombination of the second plasmid at the first integration site [55]. Such tandem-repeat copies make it more difficult to clone the tagged gene $[55,56]$.

Restriction enzyme mediated integration (REMI) has been used to increase the frequency of insertion mutagenesis in fungi and to increase the frequency of single-copy integrations (reviewed in [57]). REMI involves transforming protoplasts or spores with a linearised plasmid and the restriction endonuclease (RE) used to linearise the vector. The RE gains access to the nucleus and induces double stranded breaks in the genome. The ends of these breakages recombine with the linearised plasmid, integrating the vector into the genome at the recognition site of the RE [58].

By increasing the target sites for recombination, REMI usually improves the overall transformation rate and frequency of single copy integration events [49, 55, 59-64] although that is not always the case [50,65]. Conditions of transformation contribute significantly to the variable success rate of REMI. Although the enzyme type and concentration have an important impact on the number of transformants and the frequency of single-copy integrations, there are no clear rules for selecting either $[56,64,66]$, and the 
concentration of RE that produces the maximum number of transformants is enzyme and host dependent [66-68]. REMI can give rise to a significant number of different integration events including single insertion with deletion of flanking RE sites, ectopic integration in the absence of an appropriate RE site, tandem insertion and large genome deletions or inversions [50, 56, 64, 65, 68, 69]. The high frequency with which the mutant phenotype is not linked to the integrated DNA greatly complicates the use of this system for gene discovery.

\section{T-DNA tagging}

T-DNA tagging is a form of insertional mutagenesis that relies on AMT to mutate the recipient genome at random sites by integration of T-DNA carrying a selectable marker (reviewed in [1]). T-DNA tagging has been used successfully to find new genes $[30,70]$ and T-DNA tagging projects on fungi have recently been initiated in many laboratories around the world $[31,68]$. AMT is very suitable for insertional mutagenesis as it can cause a relatively high frequency of transformation [71-73] and often creates single-copy integrations [27, 31]. Also, T-DNA appears to integrate approximately at random $[31,73]$, although integration may be targeted towards transcribed regions $[70$, 74] and promoters in particular [70]. Retrieval of genomic regions flanking the T-DNA is facilitated by the general lack of major truncation or rearrangement of the T-DNA, although small deletions can occur at both borders of the T-DNA. Vector sequence from outside the T-DNA may also be present $[31,75,76]$.

The frequency of single-copy integrations can be quite variable $[27,31,73,76,77]$. Altering the frequency of single-copy integrations can be achieved by inducing or not inducing the Agrobacterium with acetosyringone prior to co-cultivation and by increasing the duration of co-cultivation [75]. The effect of induction varies between fungi, with induction causing either higher [31] or lower $[73,75]$ frequencies of single-copy integrations. A longer period of co-cultivation may also lead to a lower frequency of single-copy transformants [75].

In plants and fungi, AMT has been shown to cause mutations that are unlinked to the site of T-DNA integration $[70,78,79]$. The majority of AMT transformants may contain small ( $\sim 100$ bp) genomic deletions [79]. Even with single-copy T-DNA integrations, small or large genomic rearrangements are frequently observed. These include major chromosomal translocations in a significant proportion of tagged mutants [78, 79]. For this reason it is important that putatively T-DNA tagged mutants be tested to see if the T-DNA insertion site is linked to the mutant phenotype.

\section{Promoter and enhancer trapping}

Creating loss-of-function mutants through random insertion has several limitations. Mutations in essential or redundant genes may not be recovered and loss-of-function provides relatively unsubtle information about the mutated gene. Vectors for random insertional mutagenesis may be improved by the addition of elements that allow the detection of promoter activity or increase the transcription of contiguous genes. Such strategies are commonly used in plants [80-82] and have been used successfully on filamentous fungi with AMT and REMI [57, 83, 84]. Such a strategy will still pick up loss-of-function mutants but it will also pick up additional mutants that may be of interest.

For enhancer traps, a reporter gene, such as $g f p$ (green fluorescent protein), downstream from a minimal promoter that is insufficient for its expression, is positioned near the end of the transferred DNA. Insertion of the DNA near an enhancer element will allow expression of the reporter gene from the minimal promoter. Similarly, a promoter-less reporter gene can be used to tag promoters and monitor their expression. In a gene trap the reporter gene has a 5' splice acceptor sequence, and is only expressed when integrated into introns. Additionally, gain-of-function mutants can be generated and tagged by placing a strong promoter near the end of the transferred DNA in the orientation that will cause over-expression of flanking genes. It is possible to include combinations of these functions on one random mutagenesis construct [85].

\section{Transposon tagging}

Tagged mutations can also be produced by movement of mobile genetic elements (such as transposons) within the genome of the fungus. For a comprehensive review of transposable elements in filamentous fungi see Daboussi and Capy 2003 [86]. Transposon tagging can rely on endogenous transposons or highly active, engineered transposons can be transferred into heterologous hosts. The main advantage of a transposon mutagenesis strategy is that a high efficiency transformation system is not required. Some fungi are recalcitrant to transformation and the processes involved in transformation can be time consuming and are known to create genetic mutations unlinked to the transferred DNA. As transposition is relatively free from genomic lesions and rearrangements, it might be a useful method for highthroughput mutagenesis in some systems [87].

The transposon Impala has been highly studied for use as a gene tagging element in fungi [88]. Impala transposes by a cut and paste method. It encodes a single transposase enzyme that recognizes inverted terminal repeat sequences on the transposon and mediates the excision and reintegra- 
tion of the transposon without the involvement of host proteins. Consequently, transposons like Impala can typically be used in heterologous hosts [89]. The frequency of transposition of Impala can vary quite widely between strains, possibly dependant on the genomic position of the element [90]. While Impala does not have a reintegration hotspot within the genome (it reintegrates at a TA dinucleotide) and can excise from one chromosome and reintegrate into a different chromosome, it is not known to what extent reintegration is random $[87,90]$. Transposition does not always result in reintegration and the frequency of lost elements can be quite high ( $10-30 \%$ for Impala $[90,91])$. Rarely, genomic rearrangements can result from aberrant transposition [92].

Transposons have been used successfully to mutate and tag unknown fungal genes. A homologue of the Neurospora crassa nitrate metabolism regulator nit4 was isolated from Tolypocladium inflatum by the endogenous transposon Restless [93]. However, usually, a transposition tagging system will consist of an ectopically integrated construct containing a marker gene inactivated by a transposon carrying a second selectable marker gene. Excision of the transposon can be selected through restoration of the first marker gene and stable reintegration can be selected through the presence of the second marker gene. Heterologous transposition of Impala has been used to identify novel essential genes in Aspergillus fumigatus [87]. In that study, as transposition frequency depends on position effect, strains were evaluated for transposition frequency. A strain with a very high transposition rate of Impala was selected and that strain was used to generate a library of over two thousands individual transposition events. Of that library, $1.2 \%$ had a copy of Impala integrated in an essential gene. Impala has also been used successfully to tag genes in A. nidulans and Magnaporthe grisea [94, 95].

\section{Targeted gene disruption/replacement}

If a gene of interest has been identified, one strategy to investigate its function is to disrupt its expression and determine whether the phenotype is altered. In fungi, targeted gene disruption or replacement (knockout) can be achieved by homologous recombination. For gene disruption, the fungus is transformed with a disruption cassette consisting of sequences from the target gene flanking a selectable marker gene. The cassette is then inserted into the recipient genome at the target gene by homologous recombination. Homologous recombination can also be used to replace part of the endogenous gene, with exogenous sequences, for example promoter exchange. Recovered transformants will contain either, or both, DNA integrated by homologous recombination or by ectopic, illegitimate integration.
The efficiency of gene targeting in filamentous fungi is dependent on the length of the homologous sequence, the extent of homology, the transformation method and the genomic position of the target gene [2, 96]. Unlike homologous recombination in S. cerevisiae, where about $100 \mathrm{bp}$ of homologous sequence is often sufficient, filamentous fungi generally require at least $1 \mathrm{~kb}$ of homologous sequence, and in some cases much more for gene targeting at a practical frequency [97-99]. For efficient gene targeting, the level of homology between the target sequence and the homologous region of the transferred DNA needs to be very high, almost $100 \%$, and sequence variation may even preclude recombination of homologous sequences from different strains [2].

Gene targeting by homologous recombination in filamentous fungi has been achieved by all the transformation methods described. Although there are few comparisons available, it appears that AMT and particle bombardment may be particularly suited to homologous recombination [2]. AMT appears to be relatively efficient, often leading to a high frequency of gene targeting mediated by fungal recombination/repair proteins $[100,101]$. The generally higher frequency of recombination achieved by AMT may be due to the fact that the T-DNA is linear and single-stranded, a preferred substrate for homologous recombination [72, 102], and is accompanied by Agrobacterium proteins that specifically interact with DNA-associated proteins in the recipient cell [74]. Electroporation, has also been used successfully for gene targeting although the frequency of homologous recombination is lower than AMT $[2,103]$.

Where the frequency of homologous recombination is low, it may be necessary to screen large numbers of transformants to find those with targeted gene integration. A positive and negative selection system, whereby ectopic integration results in non-viable transformants, can be used to enrich for the targeted events sought. A gene, such as amdS which confers sensitivity to fluoro-acetamide, is included in the construct, outside the sequences homologous to the target gene [99]. Homologous recombination, but not illegitimate integration, removes the negative selection gene allowing selection of targeted gene integration. The thymidine kinase gene from the herpes simplex virus and the oliC31 mutation, which confers sensitivity to triethylin, have also been used for negative selection with significant increases in efficiency [28, 45, 104]. However, false positives were still produced in these studies through ectopic integration of only a part of the construct.

Another possible strategy for increasing the efficiency of gene targeting in fungi with a very low frequency of homologous recombination is to increase the expression of genes involved in homologous recombination [105] or decrease the expression of genes involved in nonhomologous 
end-joining (NHEJ) [106]. Homologous recombination in S. cerevisiae is dependent on the RAD51 protein [107]. In $A$. nidulans, over expression of the rad51 homologue $u v s C$ resulted in an improvement in targeting efficiency through homologous recombination [105]. However, elevated expression of $u v s C$ also suppressed colony growth rate. It has also been suggested that high levels of UVSC protein could potentially increase the frequency of homeologous recombination, leading to increased genomic instability [105]. Knockout of mus-51 and mus-52 genes required for NHEJ in $N$. crassa, allowed a frequency of homologous recombination of $100 \%$. N. crassa mus-51 and mus-52 mutants did not show defects in growth or morphology, suggesting that this might be a viable strategy.

\section{High throughput gene targeting strategies}

There is a growing need for more efficient ways to achieve targeted gene disruption, amenable to high throughput systems, in order to make use of the plethora of information yielded by fungal genome sequencing projects. In S. cerevisiae, where homologous recombination can be achieved with very short homologous sequences, PCR has been used as a quick method to generate gene targeting cassettes (reviewed in [108]). Coupled with complete genome sequence data and direct DNA transfer, this development allows a high throughput approach to investigating gene function without the need for cloning. With some modifications, this approach is now being applied to filamentous fungi.
Overlap or fusion PCR is a rapid method for creating gene targeting cassettes with large $(>1 \mathrm{~kb})$ homologous regions suitable for recombination in filamentous fungi (Figure 1). PCR is first used to amplify the flanking regions of the targeted gene and to amplify a selectable marker cassette. The primer for one segment has a tail complementary to the primer used to amplify the adjacent segment of the final product. The first round products are generally gel purified and mixed in equimolar ratios, then reamplified using primers complementary to each end of the final product [109]. This method has been used to create constructs for gene replacement in A. awamori [99] and for targeted in vivo promoter exchange [110]. A variation of the technique, in which overlapping segments were present on both primers at a join (termed double-jointed PCR), was used to create linear constructs for biolistic transformation of Cryptococcus neoformans [111] and for protoplast transformations of $A$. nidulans, A. fumigatus and Fusarium graminarium [112].

Another rapid method of creating linear gene disruption constructs is ligation-mediated PCR. The flanking regions of the target gene are amplified using PCR primers which include restriction sites compatible with those in the selection cassette. Purified products are digested with restriction enzymes and directionally ligated to the digested selection cassette, then the desired product is gel purified and amplified [113]. This method has an advantage over overlap PCR in that the selection cassette is not reamplified so has less opportunity to accumulate mutations that may disable it. Additionally, the number and size of primers required
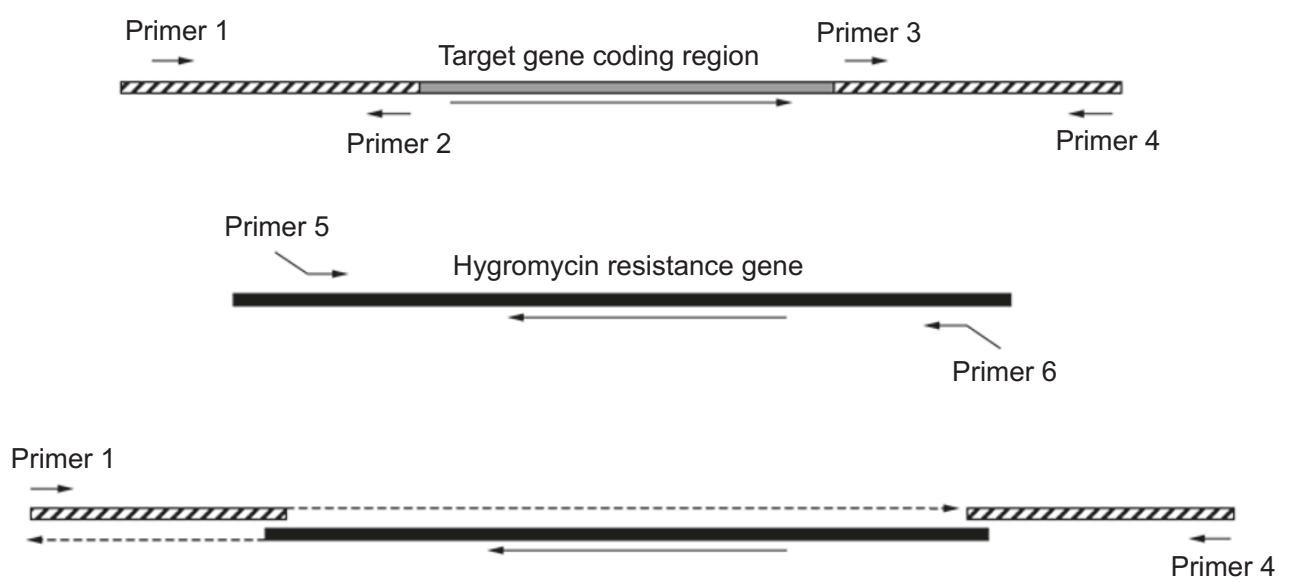

Figure 1 Creation of a gene replacement construct by overlap PCR. The flanking regions $(=1 \mathrm{~kb})$ of the target gene are amplified using primers 1-4. The selectable marker cassette, including constitutive promoter and terminator sequences, is amplified using primers 5 and 6, which have tails complementary to primers 2 and 3 respectively. PCR products are gel purified and mixed at equal molarity. During the final PCR the overlapping sections allow the fragments to act as primers for each other, and the whole construct is amplified using primers 1 and 4. If the product is to be cloned, restriction sites can be included in tails on primers 1 and 4 . A long template polymerase such as Expand Long Template (Roche) is required. 
is reduced. The use of these PCR methods to create linear constructs is particularly suited to direct DNA transfer methods such as particle bombardment or protoplast transformation. Gene targeting cassettes created by PCR can also be inserted into binary vectors for use in AMT [99].

\section{In vitro transposon tagging}

Gene discovery and the creation of gene disruption constructs can be performed simultaneously by transposon arrayed gene knockout (TAGKO) [114]. In TAGKO, a cosmid library is created from the whole fungal genome (Figure 2). Transposon Tn7 carrying a selectable marker gene integrates, by an enzyme-catalysed (transposase) in vitro reaction, into a pool of cosmids or a single cosmid, randomly disrupting genes. Sequencing primed by transposon sequences identifies disrupted genes, allowing selection of cosmids carrying disruptions in genes of interest, for gene disruption by homologous recombination. Restriction digestion of selected cosmids releases a linear insert suitable for transformation using protoplast or electroporation methods, and phenotypic characterisation of disruption mutants can then follow. The process can be used to create a range of mutations in a single gene or to disrupt genes from throughout the genome.
TAGKO has been used successfully for targeted gene disruption [103, 115]. Electroporation of 11 TAGKO constructs into Mycosphaerella graminicola protoplasts produced disruption mutants at a targeting frequency of $15-28 \%$, which is very high for that method of transformation [103]. The high frequency of gene targeting probably reflects the length of homologous regions in TAGKO constructs ( $\sim 40 \mathrm{~kb})$ [103]. However, much higher frequencies of homologous recombination (44\%) can be achieved in M. graminicola by AMT [101]. In vivo recombination has been used to rapidly convert BACs to binary vectors [116], conversion of TAGKO cosmids to binary vectors might create a very efficient gene targeting system based on TAGKO-AMT [103].

In a system similar to TAGKO, the GPS-1 genome-priming system (New England Biolabs) has been used to create gene targeting constructs. In this system, a gene of interest is ligated into the $\mathrm{A} / \mathrm{T}$ cloning vector pGEM (Promega) [117]. In vitro transposition of the pGPS1 transprimer 1 transposon, modified to carry the selectable marker gene pyr4, is used to randomly mutate the gene of interest. The constructs are amplified in E. coli, the transposon disruption is mapped, and the vector is linearized ready for direct DNA transfer to the target fungus.

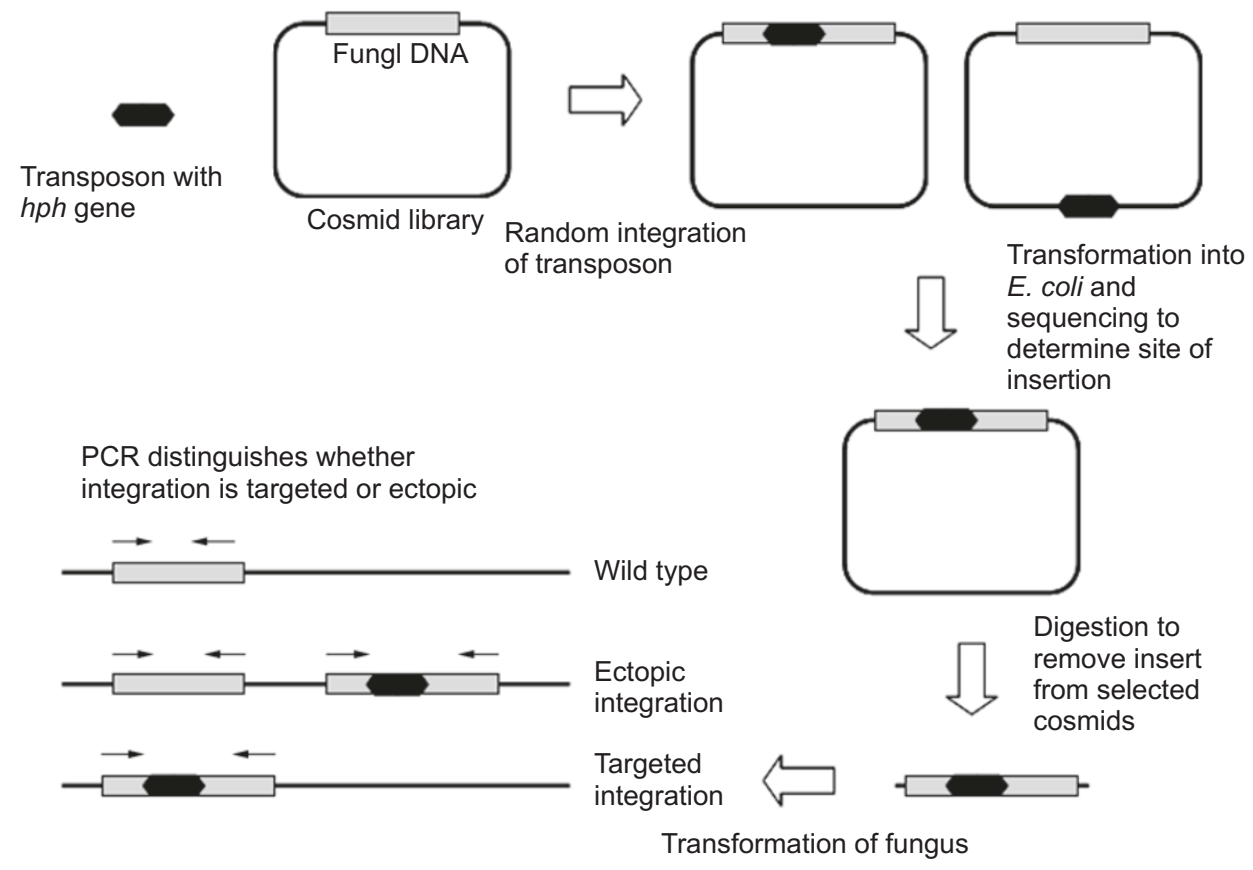

Figure 2 Transposon arrayed gene knockout (TAGKO). The transposon integrates randomly into cosmids in vitro. Mutated cosmids are transformed into E. coli, and the transposon insertion sites are determined by sequencing adjacent to the transposon. Selected cosmids are digested with endonucleases and the linear fragment is used for fungal transformation. Ectopic and targeted integration can be distinguished by PCR. 


\section{Laboratory information management}

With whole genome sequencing and advances in high throughput gene disruption, mutagenesis and phenotypic characterization of an entire organism have become possible. In many systems, logistical rather than technical difficulties limit this approach. The creation and analysis of many thousands of strains require careful coordination and standardisation both of experimental steps and in data collection. PACLIMS (Phenotype Assay Component Laboratory Information Management System) is an online data management system that was designed to support the multi-institutional production of fifty thousand random insertion mutants of $M$. grisea [118]. PACLIMS uses barcoding to track the biological material and control the analysis through a carefully planned protocol. PACLIMS builds up an online, searchable database and is open source software that is currently freely available from: http://paclims.sourceforge.net.

\section{Post-transcriptional gene silencing}

Targeted gene disruption or replacement (knockout), as described above, has been the technique of choice for creating null mutants of specific genes in many fungi. Gene knockout often requires significant length of homologous sequence for integration and even then, the frequency of targeted integration can be very low in some systems. Further, the consequent phenotype may be lethal or it may not be observed in heterokaryotic tissue. An alternative method, that overcomes some of the problems associated with knockouts, is to disrupt gene expression at a post-transcription level (gene knockdown) by targeting the mRNA rather than the gene.

RNA interference (RNAi) is a sequence-specific post-transcriptional gene-silencing phenomenon [119]. RNAi can be induced by the presence of foreign nucleic acid sequences such as RNA viruses and transgenes. An essential, rate-limiting step is the formation of dsRNA which is cleaved by dicer to produce small interfering RNA (siRNA) [120, 121]. mRNA with sequence identity to the siRNA is specifically degraded by an RNA-induced silencing complex (RISC) [122]. In some organisms, an RNA-dependent RNA polymerase (RdRP) uses antisense siRNA to prime the conversion of endogenous mRNA into dsRNA amplifying the silencing signal [120]. RNAi has recently been used as a functional genomics tool in fungi. However, RNAi is still a relatively undeveloped technique that does not always reduce expression to a point where a phenotype change is seen.

RNAi has been used successfully for gene knockdown in filamentous fungi [3]. In its most effective form, an ectopi- cally integrated RNAi construct codes for double stranded RNA, identical to part of the endogenous sequence being targeted [120, 123-125]. The linear RNAi knockdown transcript contains an inverse repeat of the target sequence that folds up into a "hairpin" in which the inverse repeat is double stranded. The repeat sequences are typically about $500 \mathrm{bp}$ of coding sequence separated by a spacer sequence of about $250 \mathrm{bp}$ [126]. A substantial decrease in efficiency was found when using repeat sequences of $200 \mathrm{bp}$ rather than $600 \mathrm{bp}$ or $900 \mathrm{bp}$ [125]. The inclusion of an intron in the spacer region may greatly increase the silencing efficiency, possibly through enhanced export of the hairpin from the nucleus during splicing [125].

RNAi silencing vectors for rapid cloning of target sequences are available [127]. High throughput cloning of target sequences for RNAi in filamentous fungi can now also be achieved using Gateway ${ }^{\circledR}$ (Invitrogen) in vitro recombination technology. In this system, site-specific recombination directs the incorporation of PCR amplified target sequences into an intron-containing, RNAi expression cassette (developed by Ellen Fox, Don Gardiner and Barbara Howlett, unpublished).

Gene knockdown has some advantages when compared to gene knockout. Knockdown requires relatively short stretches of sequence information. This is a major advantage for organisms for which there is little sequence information. As RNAi works at the mRNA level, its efficacy is not compromised by the presence of non-transformed nuclei or multi-copy genes due to aneuploidy. Where there is some sequence conservation between genes, one or a few RNAi constructs can be used to knockdown an entire family of genes. This ability can be especially useful where gene redundancy would otherwise mask the phenotype of a gene knockout. To avoid the problem of lethal knockouts, RNAi can be used to achieve down regulation or conditional promoters can be used to control knockdown [125].

There are also some limitations to RNAi. Firstly, RNAi usually does not abolish expression of the target gene and the extent of knockdown of transcript is highly variable making it necessary to screen transformants for high levels of knockdown [124, 126]. Also, the threshold of expression knockdown required before a change in phenotype is observed varies between target genes. This problem can be mitigated to some extent by simultaneously targeting a second gene, as a control, by incorporating two target sequences in a chimeric RNAi hairpin construct $[41,126]$. For this strategy, the second gene could be a transgenic reporter gene such as $g f p$. The simultaneous silencing of the reporter and endogenous gene would also be amenable to high throughput genetic analyses [41].

Another potential disadvantage is that, as only a short sequence is required for RNAi, genes other than those 
targeted might be silenced. Testing for the possibility of off-target effects is simpler for organisms for which there is a complete genome sequence. Transcripts for those sequences that are most similar can be assessed for down regulation, to determine off target effects. In addition, integration of the silencing construct might directly influence the phenotype by randomly knocking out genes. Several knockdown transformants displaying the same phenotype adequately demonstrate that this has not occurred. Finally, knockdown transformants can not be complemented as the endogenous gene itself has not been altered. It is possible to overcome this by re-transformation with a full gene sequence that has the codons altered to avoid targeting by the RNAi construct. This concept is now more achievable with the ability to obtain synthetic gene constructs. Also, an RNAi complementation method for mammalian cells has been achieved by knockdown using small hairpin RNA targeting untranslated regions (UTR) of the endogenous gene with complementation by a simultaneously expressed gene lacking the UTRs [128]. Alternatively, an inducible promoter driving expression of the RNAi construct can be used [126].

\section{Other targeted gene strategies}

Although the targeted creation of loss-of-function mutants is the most common method for examining gene function, other methods have also been applied to filamentous fungi. Most of these methods depend upon modifying the gene in vitro and then transforming it, either into the same or a different genetic background. The advantage of these techniques is that they provide specific additional information that is not obtainable through lossof-function mutation. Introducing the gene into a different genetic background such as into yeast or E. coli, allows the gene and its product to be examined in isolation from endogenous proteins. Genes can also be examined within a specific, defined context. For example, the function and activity of fungal pathogenicity genes have been examined by transferring the genes to a host plant and expressing them in planta [129] for example, functional cloning of avr genes from Cladosporium fulvum has been achieved by expressing a cDNA library in tomato [130]. Similarly, the function of fungal fungicide resistance genes can be examined by expressing them in a sensitive strain of the same fungus [131].

The yeast one-hybrid system has been used to isolate transcriptional activators of promoters from Trichoderma reesei $[132,133]$. In two studies, a Trichoderma promoter has been cloned into a yeast replication vector upstream from a minimal promoter/His 3 selective marker gene cassette then transformed into yeast. In a second round of trans- formation, the yeast were transformed with a Trichoderma cDNA expression library and transformants expressing the His 3 marker gene were isolated on the assumption that His 3 expression was triggered by a cDNA transcription factor binding to the Trichoderma promoter. Transcription factors that control specific Trichoderma gene expression have been successfully retrieved from yeast transformants by this method $[132,133]$.

The interaction between two specific fungal gene products has been examined in the yeast two-hybrid system $[134,135]$. In this system, the two genes are each cloned as fusions to either the activation domain or the binding domain of the GAL4 transcription factor on a yeast replicative vector and transferred into $S$. cerevisiae. Transcription activation of GAL4-activated reporter genes requires that the two GAL4 domains be brought together by specific interaction between the two proteins.

Where a cloned gene of interest is transformed for ectopic integration into the same species and strain it was originally retrieved from, its function is usually examined either through over-expression [37, 136, 137], or though transcription or translation fusions to marker genes such as $g f p$ [138]. For over-expression studies, the coding region of the gene of interest can be cloned downstream from a strong heterologous promoter, or under the control of an inducible promoter such as alcA so there is some control over the level of over-expression [136, 139].

Enhancer and promoter elements can also be engineered and reintroduced ectopically to investigate their function. Transcriptional fusions to reporter genes have been used to define cis-acting promoter elements through the construction of a series of systematic modifications made to the promoter prior to its integration and expression in the host genome [140]. The effect of each mutation can be measured by changes in expression of the reporter gene. One problem with this approach is that the promoter may not be integrated into the same genetic context it was originally in and this could affect its expression [140].

\section{From genomics to proteomics}

There is an increasing interest in looking beyond the genome and investigating the functions and interactions of the proteins themselves. By using recombinant DNA approaches to fluorescently label endogenous proteins (e.g. GFP fusion proteins) or for epitope-tagging (translational fusion of an antigenic determinant to the protein of interest) the resources developed for studying the genome are now being used to study the proteome. Combining annotated sequence information with the method of targeted DNA integration achieved through homologous recombination, a library of yeast with an epitope-tag integrated at the 
C-terminus of nearly every open reading frame has been created [141]. Although large-scale epitope-tagged libraries have not been made for any filamentous fungi, targeted epitope-tagging using high-throughput PCR methods have been achieved in at least one filamentous fungus [142].

High-throughput immunoprecipitation experiments with epitope-tagged libraries make it possible to look beyond transcription profiles and directly monitor protein location, post-transcriptional modifications and interactions with other proteins. Further, by creating fusions with fluorescent proteins such as GFP and applying advanced techniques such as fluorescence resonance energy transfer it is now possible to detect specific conformational changes within proteins [143]. In future we may see tagged proteins become widely used to study biochemical reactions in vivo, in real time.

\section{Summary}

Although a robotic, mass data collection approach may not be the inevitable endpoint of functional genomics in all filamentous fungi, it is clear that a major trend is towards high throughput strategies for whole genome analysis. That outcome can now be achieved in model systems by using entire genome sequences and linking rapid constructbuilding techniques, efficient transformation systems and phenotype assays supported by high throughput data management systems.

For those who are developing functional genomics in more recalcitrant fungi, there are many different approaches that can be taken. While REMI is still being used successfully for random mutagenesis, there is a clear trend away from REMI towards using T-DNA tagging for this purpose. T-DNA tagging generally appears to have a higher transformation frequency and is thought to create fewer genomic lesions that are unlinked to the DNA insertion site, although these do still occur. As a strategy for insertional mutagenesis, in vivo transposon tagging appears to be theoretically the most ideal approach. It does not require repeated transformation and to some extent it avoids the problems associated with mutations that are not linked to the tagged site. However, transposon tagging has not been taken up widely for fungal research, probably due to a perception that it lacks efficiency and also because it is more complicated to initially set up than a transformation-based mutation system. Similarly, the extra steps required to set up in vitro transposon tagging may be a barrier to the uptake of strategies such as TAGKO for random mutagenesis.

A major problem for both random mutagenesis and targeted gene disruption is the multinucleate nature of filamentous fungi. RNAi techniques may offer a solution to this problem, at least for gene targeting. However, RNAi is a relatively new technique that as yet has not been thoroughly developed for use in filamentous fungi, and does not work efficiently in every system. As RNAi has not been developed as a random mutagenesis strategy, something that seems technically feasible, the multinucleate state remains a problem for generating random, loss-of-function, mutants. It is surprising then, that more use has not been made of enhancer-traps and similar devices that extend the range of mutations that can be detected by insertional mutagenesis.

All the techniques described here have advantages and limitations and each may be more suitable in one system and less suitable in another. The particular approach taken will be dictated to some extent by laboratory-specific practices, by the practicalities of the individual organisms and the outcome that is desired. However, systematic planning is needed from an early stage to take advantage of the best developments for efficient experimentation that are now available.

\section{Acknowledgements}

The authors thank Professor Barry Scott of Massey University, New Zealand and Simon Bulman from New Zealand Institute for Crop and Food Research Ltd for critically reviewing the manuscript. The writing of this review was supported by a grant from the New Zealand Tertiary Education Commission, grant application number 02-LIN-501.

\section{References}

1 Michielse CB, Hooykaas PJJ, van den Hondel CAMJJ, Ram AFJ. Agrobacterium-mediated transformation as a tool for functional genomics in fungi. Curr Genet 2005; 48:1-17.

2 Davidson RC, Cruz MC, Sia RAL, et al. Gene disruption by biolistic transformation in serotype D strains of Cryptococcus neoformans. Fungal Genet Biol 2000; 29:38-48.

3 Nakayashiki H. RNA silencing in fungi: mechanisms and applications. FEBS Lett 2005; in press.

4 Mullins ED, Kang S. Transformation: a tool for studying fungal pathogens of plants. Cell Mol Life Sci 2001; 58:2043-52.

5 Ruiz-Diez B. Strategies for the transformation of filamentous fungi. J Appl Microbiol 2002; 92:189-95.

6 Lorito M, Hayes CK, Di Pietro A, Harman GE. Biolistic transformation of Trichoderma harzianum and Gliocladium virens using plasmid and genomic DNA. Curr Genet 1993; 24:349-56.

7 Ballance DJ, Buxton FP, Turner G. Transformation of Aspergillus nidulans by the ornithine-5'-phosphate decarboxylase gene of Neurospora crassa. Biochem Biophys Res Commun 1983; 112:284-9.

8 Fitzgerald AM, Mudge AM, Gleave AP, Plummer KM. Agrobacterium and PEG-mediated transformation of the phytopathogen Venturia inaequalis. Mycol Res 2003; 107:803-10. 
9 Kuo CY, Chou SY, Huang CT. Cloning of glyceraldehyde-3phosphate dehydrogenase gene and use of the $g p d$ promoter for transformation in Flammulina velutipes. Appl Microbiol Biotechnol 2004; 65:593-9.

10 Hazell BW, Teo VSJ, Bradner JR, Berquist PL, Nevalainen, K.M.H. Rapid transformation of high secreting mutant strains of Trichoderma reesei by microprojectile bombardment. Lett Appl Microbiol 2000; 30:282-6.

11 Vijayaraghavan Y, Kapoor M. Disruption of the NAD(+)-specific glutamate dehydrogenase gene of Neurospora crassa by means of the RIP (repeat-induced point mutations) process. Biochem Cell Biol 1996; 74:29-40.

12 Ozeki K, Kyoya F, Hizume K, et al. Transformation of intact Aspergillus niger by electroporation. Biosci Biotechnol Biochem 1994; 58:2224-7.

13 Ward M, Kodama KH, Wilson LJ. Transformation of Aspergillus awamori and A. niger by electroporation. Experimental Mycol 1989; 13:289-93.

14 Ruiz-Diez B, Martinez-Suarez JV. Electroporation of the human pathogenic fungus Scedosporium prolificans mediated by repeditive rDNA sequences. FEMS Immunol Med Microbiol 1999; 25:275-82.

15 Reyes I, Bernier L, Antoun H. Rock phosphate solubilization and colonization of maize rhizosphere by wild and genetically modified strains of Penicillium rugulosum. Microb Ecol 2002; 44:39-48.

16 Te'o VS, Bergquist PL, Nevalainen KM. Biolistic transformation of Trichoderma reesei using the Bio-Rad seven barrels Hepta Adaptor system. J Microbiol Methods 2002; 51:393-9.

17 Parker DM, Hilber UW, Bodmer M, et al. Production and transformation of conidia of Venturia inaequalis. Phytopathology 1995; 85:87-91.

18 Cvitanich C, Judelson HS. Stable transformation of the oomycete, Phytophthora infestans, using microprojectile bombardment. Curr Genet 2003; 42:228-35.

$19 \mathrm{Yu}$ JJ, Cole GT. Biolistic transformation of the human pathogenic fungus Coccidioides immitis. J Microbiol Methods 1998; 33:12941.

20 Barreto CC, Alves LC, Aragao FJL, et al. High frequency gene transfer by microprojectile bombardment of intact conidia from the entomopathogenic fungus Paecilomyces fumosoroseus. FEMS Microbiol Lett 1997; 156:95-9.

21 Bogo MR, Vainstein M H, Aragao FJL, Rech E, Schrank A. High frequency gene conversion among benomyl resistant transformants in the entomopathogenic fungus Metarhizium anisopliae. FEMS Microbiol Lett 1996; 142:123-27.

22 Forbes PJ, Millam S, Hooker JE, Harrier LA. Transformation of the arbuscular mycorrhiza Gigaspora rosea by particle bombardment. Mycol Res 1998; 102:497-501.

23 Durand R, Rascle C, Fischer M, Fevre M. Transient expression of the beta-glucuronidase gene after biolistic transformation of the anaerobic fungus Neocallimastix frontalis. Curr Genet 1997; 31:158-61.

24 Sunagawa M, Magae Y. Transformation of the edible mushroom Pleurotus ostreatus by particle bombardment. FEMS Microbiol Lett 2002; 211:143-6.

25 Viterbo A, Haran S, Friesem D, Ramot O, Chet I. Antifungal activity of a novel endochitinase gene (chit36) from Trichoderma harzianum Rifai TM. FEMS Microbiol Lett 2001; 200:169-74.
26 Idnurm A, Reedy JL, Nussbaum JC, Heitman J. Cryptococcus neoformans virulence gene discovery through insertional mutagenesis. Eukaryot Cell 2004; 3:420-9.

27 Meyer V, Mueller D, Strowig T, Stahl U. Comparison of different transformation methods for Aspergillus giganteus. Curr Genet 2003; 43:371-7.

28 Khang CH, Park SY, Lee YH, Kang S. A Dual selection based, targeted gene replacement tool for Magnaporthe grisea and Fusarium oxysporum. Fungal Genet Biol 2005; 42:483-92.

29 Zeilinger S. Gene disruption in Trichoderma atroviride via Agrobacterium-mediated transformation. Curr Genet 2004; 45:54-60.

30 Li M, Gong X, Zheng J, et al. Transformation of Coniothyrium minitans, a parasite of Sclerotinia sclerotiorum, with Agrobacterium tumefaciens. FEMS Microbiol Lett 2005; 243:3239.

31 Combier JP, Melayah D, Raffier C, Gay G, Marmeisse R. Agrobacterium tumefaciens-mediated transformation as a tool for insertional mutagenesis in the symbiotic ectomycorrhizal fungus Hebeloma cylindrosporum. FEMS Microbiol Lett 2003; 220:141-8.

32 Vijn I, Govers F. Agrobacterium tumefaciens mediated transformation of the oomycete plant pathogen Phytophthora infestans. Mol Plant Pathol 2003; 4:459-67.

33 Sullivan TD, Rooney PJ, Klein BS. Agrobacterium tumefaciens integrates transfer DNA into single chromosomal sites of dimorphic fungi and yields homokaryotic progeny from multinucleate yeast. Eukaryot Cell 2002; 1:895-905.

34 Davis RH. Adaptation in pantothenate-requiring Neurospora. II. Nuclear competition during adaptation. Amer J Bot 1960; 47:648-54.

35 Rolland S, Jobic C, Fevre M, Bruel C. Agrobacterium-mediated transformation of Botrytis cinerea, simple purification of monokaryotic transformants and rapid conidia-based identification of the transfer-DNA host genomic DNA flanking sequences. Curr Genet 2003; 44:164-71.

36 Weiland JJ. Transformation of Pythium aphanidermatum to geneticin resistance. Curr Genet 2003; 42:344-52.

37 Rocha-Ramirez V, Omero C, Chet I, Horwitz BA, Herrera-Estrella A. Trichoderma atroviride G-protein a-subunit gene tgal is involved in mycoparasitic coiling and conidiation. Eukaryot Cell 2002; 1:594-605.

38 Robinson M, Sharon A. Transformation of the bioherbicide Colletotrichum gloeosporioides $f$. sp. aeschynomene by electroporation of germinated conidia. Curr Genet 1999; 36:98-104.

39 Peterbauer CK, Litscher D, Kubicek CP. The Trichoderma atroviride sebl (stress response element binding) gene encodes an AGGGG-binding protein which is involved in the response to high osmolarity stress. Mol Genet Genomics 2002; 268:22331.

40 Chen C, Harel A, Gorovoits R, Yarden O, Dickman MB. MAPK regulation of sclerotial development in Sclerotinia sclerotiorum is linked with $\mathrm{pH}$ and cAMP sensing. Mol Plant Microbe Interact 2004; 17:404-13.

41 Fitzgerald A, van Kan JAL, Plummer KM. Simultaneous silencing of multiple genes in the apple scab fungus, Venturia inaequlis, by expression of RNA with chimeric inverted repeats. Fungal Genet Biol 2004; 41:963-71.

42 Wirsel SGR, Voegele RT, Banninger R, Mendgen KW. Cloning 
of beta-tubulin and succinate dehydrogenase genes from Uromyces fabae and establishing selection conditions for their use in transformation. Eur J Plant Pathol 2004; 110:767-77.

43 Temporini ED, Alvarez ME, Mautino MR, Folco HD, Rosa AL. The Neurospora crassa cfp promoter drives a carbon sourcedependent expression of transgenes in filamentous fungi. J Appl Microbiol 2004; 96: 1256-64.

44 Chung KR, Shilts T, Li W, Timmer LW. Engineering a genetic transformation system for Colletotrichum acutatum, the causal fungus of lime anthracnose and postbloom fruit drop of citrus. FEMS Microbiol Lett 2002; 213:33-9.

45 Takahashi T, Hatamoto O, Koyama Y, Abe K. Efficient gene disruption in the koji-mold Aspergillus sojae using a novel variation of the positive-negative method. Mol Gen Genomics 2004; 272:344-52.

46 d'Enfert C. Selection of multiple disruption events in Aspergillus fumigatus using the orotidine-5'-decarboxylase gene, pyrG, as a unique transformation marker. Curr Genet 1996; 30:76-82.

47 Hartl L, Seiboth B. Sequential gene deletions in Hypocrea jecorina using a single blaster cassette. Curr Genet 2005; 48:20411.

48 Staab JF, Sundstrom P. URA3 as a selectable marker for disruption and virulence assessment of Candida albicans genes. Trends Microbiol 2003; 11:69-73.

49 Cummings WJ, Celerin M, Crodian J, Brunick LK, Zolan ME. Insertional mutangenesis in Coprinus cinereus: use of a dominant selectable marker to generate tagged, sporulation-defective mutants. Curr Genet 1999; 36:371-82.

50 Chung KR, Ehrenshaft M, Wetzel DK, Daub ME. Cercosporindeficient mutants by plasmid tagging in the asexual fungus $\mathrm{Cer}$ cospora nicotianae. Mol Gen Genomics 2003; 270:103-13.

51 Combier JP, Melayah D, Raffier C, et al. Nonmycorrhizal (Myc(-)) mutants of Hebeloma cylindrosporum obtained through insertional mutagenesis. Mol Plant Microbe Interact 2004; 17:1029-38.

52 Seong K, Hou Z, Tracy M, Kistler HC, Xu JR. Random insertional mutagenesis identifies genes associated with virulence in the wheat scab fungus Fusarium graminearum. Phytopathology 2005; 95:744-50.

53 Moriwaki A, Kihara J, Kobayashi T, et al. Insertional mutagenesis and characterization of a polyketide synthase gene (PKS1) required for melanin biosynthesis in Bipolaris oryzae. FEMS Microbiol Lett 2004; 238:1-8.

54 Weidner G, D’Enfert C, Koch A, Mol PC, Brackhage AA. Development of a homologous transformation system for the human pathogenic fungus Aspergillus fumigatus based on the pyrG gene encoding orotidine 5'-monophosphate decarboxylase. Curr Genet 1998; 33:378-85.

55 Itoh Y, Scott B. Effect of de-phosphorylation of linearized pAN71 and of addition of restriction enzyme on plasmid integration in Penicillium paxilli. Curr Genet 1997; 32:147-51.

56 Brown JS, Aufauvre-Brown A, Holden DW. Insertional mutagenesis of Aspergillus fumigatus. Mol Gen Genet 1998; 259:32735.

57 Kahmann R, Basse C. REMI (Restriction Enzyme Mediated Integration) and its impact on the isolation of pathogenicity genes in fungi attacking plants. Eur J Plant Pathology 1999; 105:221-9.

58 Riggle PJ, Kumamoto CA. Genetic analysis in fungi using restriction-enzyme-mediated integration. Curr Opin Microbiol 1998; 1:395-9.

59 Bolker M, Bohnert, HU, Braun KH, Gorl J, Kahmann R. Tagging pathogenicity genes in Ustilago maydis by restriction enzymemediated integration (REMI). Mol Gen Genet 1995; 248:54752.

60 Redman RS, Rodriguez RJ. Factors affecting the efficient transformation of Colletotrichum species. Experimental Mycol 1994; 18:230-46.

61 Kaufman G, Horwitz BA, Hadar R, Ullmann Y, Berdicevsky I. Green fluorescent protein (GFP) as a vital marker for pathogenic development of the dermatophyte Trichophyton mentagrophytes. Microbiology 2004; 150:2785-90.

62 Brown Jr. DH, Slobodkin IV, Kumamoto CA. Stable transformation and regulated expression of an inducible reporter construct in Candida albicans using restriction enzyme-mediated integration. Mol Gen Genet 1996; 251:75-80.

63 Linnemannstons P, Voss T, Hedden P, Gaskin P, Tudzynski B. Deletions in the gibberellin biosynthesis gene cluster of Gibberella fujikuroi by restriction enzyme-mediated integration and conventional transformation-mediated mutagenesis. Appl Environ Microbiol 1999; 65:2558-64.

64 Thon MR, Nuckles EM, Vaillancourt LJ. Restriction enzymemediated integration used to produce pathogenicity mutants of Colletotrichum graminicola. Mol Plant Microbe Interact 2000; 13:1356-65.

65 Redman RS, Ranson JC, Rodriguez RJ. Conversion of the pathogenic fungus Colletotrichum magna to a nonpathogenic, endophytic mutualist by gene disruption. Mol Plant Microbe Interact 1999; 12:969-75.

66 Granado JD, Kertesz-Chaloupkova K, Aebi M, Kues U. Restriction enzyme-mediated DNA integration in Coprinus cinereus. Mol Gen Genet 1997; 256:28-36.

67 Sato T, Yaegashi K, Ishii S, et al. Transformation of the edible basidiomycete Lentinus edodes by restriction enzyme-mediated integration of plasmid DNA. Biosci Biotechnol Biochem 1998; 62:2346-50.

68 Rogers CW, Challen MP, Green JR, Whipps JM. Use of REMI and Agrobacterium-mediated transformation to identify pathogenicity mutants of the biocontrol fungus Coniothyrium minitans. FEMS Microbiol Lett 2004; 241:207-14.

69 Balhadere PV, Foster AJ, Talbot NJ. Identification of pathogenicity mutants of the rice blast fungus Magnaporthe grisea by insertional mutagenesis. Mol Plant Microbe Interact 1999; 12:129-42.

70 Walton FJ, Idnurm A, Heitman J. Novel gene functions required for melanization of the human pathogen Cryptococcus neoformans. Mol Microbiol 2005; 57:81-1396.

71 Sun CB, Kong QL, Xu WS. Efficient Transformation of Penicillium chrysogenum mediated by Agrobacterium tumefaciens LBA4404 for Cloning of Vitreoscilla hemoglobin gene. Electron J Biotechn 2002; 5:21-8.

72 de Groot MJ, Bundock P, Hooykaas PJ, Beijersbergen AG. Agrobacterium tumefaciens-mediated transformation of filamentous fungi. Nat Biotechnol 1998; 16:839-42.

73 Mullins ED, Chen X, Romaine P, et al. Agrobacterium-Mediated transformation of Fusarium oxysporum: an efficient tool for insertional mutagenesis and gene transfer. Phytopathology 2001; 91:173-80. 
74 Bako L, Umeda M, Tiburcio AF, Schell J, Koncz C. The VirD2 pilot protein of Agrobacterium transferred DNA interacts with the TATA box-binding protein and a nuclear protein kinase in plants. Proc Natl Acad Sci U S A 2003; 100:10108-13.

75 Rho HS, Kang S, Lee YH. Agrobacterium tumefaciens-mediated transformation of the plant pathogenic fungus, Magnaporthe grisea. Mol Cells 2001; 12:407-11.

76 Covert SF, Kapoor P, Lee MH, Briley A, Nairn CJ. Agrobacterium tumefaciens-mediated transformation of Fusarium cirinatum. Mycol Res 2001; 105:259-64.

77 dos Reis MC, Fungaro MHP, Duarte RTD, Furlaneto L, Furlaneto MC. Agrobacterium tumefaciens-mediated genetic transformation of the entomopathogenic fungus Beauveria bassiana. J Microbiol Methods 2004; 58:197-202.

78 Tax FE, Vernon DM. T-DNA-associated duplications/translocations in Arabidopsis. Implications for mutant analysis and functional genomics. Plant Physiol 2001; 126:1527-38.

79 Forsbach A, Schubert D, Lechtenberg B, Gils M, Schmidt R. A comprehensive characterization of single-copy T-DNA insertions in the Arabidopsis thaliana genome. Plant Mol Biol 2003; 52:161-76.

80 Peng H, Huang HC, Yang Y, et al. Functional analysis of GUS expression patterns and T-DNA integration characteristics in rice enhancer trap lines. Plant Sci 2005; 168:1571-9.

81 Springer PS. Gene traps: tools for plant development and genomics. Plant Cell 2000; 12:1007-20.

82 Mathews H, Clendennen SK, Caldwell CG, et al. Activation tagging in tomato identifies a transcriptional regulator of anthocyanin biosynthesis, modification, and transport. Plant Cell 2003; 15:1689-703.

83 Aichinger C, Hansson K, Eichhorn $\mathrm{H}$, et al. Identification of plant-regulated genes in Ustilago maydis by enhancer-trapping mutagenesis. Mol Gen Genomics 2003; 270:303-14.

84 Shuster JR, Connelley MB. Promoter-tagged restriction enzymemediated insertion (PT-REMI) mutagenesis in Aspergillus niger. Mol Gen Genet 1999; 262:27-34.

85 Jeong DH, An S, Kang HG, et al. T-DNA insertional mutagenesis for activation tagging in rice. Plant Physiol 2002; 130:163644.

86 Daboussi M-J, Capy P. Transposable elements in filamentous fungi. Ann. Rev. Microbiol 2003; 57:275-99.

87 Firon A, Villalba F, Beffa R, d'Enfert C. Identification of essential genes in the human fungal pathogen Aspergillus fumigatus by transposon mutagenesis. Eukaryot Cell 2003; 2:247-55.

88 Langin T, Capy P, Daboussi MJ. The transposable element Impala, a fungal member of the Tc1-mariner superfamily. Mol Gen Genet 1995; 246:19-28.

89 Hua-Van A, Pamphile JA, Langin T, Daboussi MJ. Transposition of autonomous and engineered Impala transposons in Fusarium oxysporum and a related species. Mol Gen Genet 2001; 264:72431.

90 Giulia M, Nicosia LD, Brocard-Masson C, et al. Heterologous transposition in Aspergillus nidulans. Mol Microbiol 2001; 39:1330-44.

91 de Queiroz MV, Daboussi M-J. Impala, a transposon from Fusarium oxysporum, is active in the genome of Penicillium griseoroseum. FEMS Microbiol Lett 2003; 218:317-21.

92 Hua-Van A, Langin T, Daboussi MJ. Aberrant transposition of a Tc1-mariner element, Impala, in the fungus Fusarium oxyspo- rum. Mol Genet Genomics 2002; 267:79-87.

93 Kempken F, Kuck U. Tagging of a nitrogen pathway-specific regulator gene in Tolypocladium inflatum by the transposon Restless. Mol Gen Genet 2000; 263:302-8.

94 Cecchetto G, Amillis S, Dianinas G, Scazzocchio C, Drevet C. The AzgA purine transporter of Aspergillus nidulans. J Biol Chem 2004; 279:3132-41.

95 Villalba F, Lebrun MH, Hua-Van A, Daboussi MJ, GrosjeanCournoyer MC. Transposon Impala, a novel tool for gene tagging in the rice blast fungus Magnaporthe grisea. Mol Plant Microbe Interact 2001; 14:308-15.

96 Bird D, Bradshaw R. Gene targeting is locus dependent in the filamentous fungus Aspergillus nidulans. Mol Gen Genet 1997; 255:219-25.

97 Michielse CB, Arentshorst M, Ram AFJ, van den Hondel CAMJJ. Agrobacterium-mediated transformation leads to improved gene replacement efficiency in Aspergillus awamori. Fungal Genet Biol 2005; 42:9-19.

98 Wilson LM, Idnurm A, Howlett BJ. Characterization of a gene (sp1) encoding a secreted protein from Leptosphaeria maculans, the blackleg pathogen of Brassica napus. Mol Plant Pathol 2002; 3:487-93.

99 Michielse CB, Arentshorst M, Ram AFJ, van den Hondel CAMJJ. Agrobacterium-mediated transformation leads to improved gene replacement efficiency in Aspergillus awamori. Fungal Genet Biol 2005; 42:9-19.

100van Attikum H, Hooykaas PJJ. Genetic requirements for the targeted integration of Agrobacterium T-DNA in Saccharomyces cerevisiae. Nucleic Acids Res. 2003; 31:826-32.

101 Zwiers LH, de Waard MA. Efficient Agrobacterium tumefaciensmediated gene disruption in the phytopathogen Mycosphaerella graminicola. Curr Genet 2001; 39:388-93.

102Bundock P, Hooykaas PJ. Integration of Agrobacterium tumefaciens T-DNA in the Saccharomyces cerevisiae genome by illegitimate recombination. Proc Natl Acad Sci U S A 1996; 93:15272-5.

103 Adachi K, Nelson GH, Peoples KA, et al. Efficient gene identification and targeted gene disruption in the wheat blotch fungus Mycosphaerella graminicola using TAGKO. Curr Genet 2002; 42:123-7.

104Gardiner DM, Howlett BJ. Negative selection using thymidine kinase increases the efficiency of recovery of transformants with targeted genes in the filamentous fungus Leptosphaeria maculans. Curr Genet 2004; 45:149-55.

105 Natsume T, Egusa M, Kodama M, et al. An appropriate increase in the transcription of Aspergillus nidulans uvs C improved gene targeting efficiency. Biosci Biotechnol Biochem 2004; 68:164956.

106Ninomiya Y, Suzuki K, Ishii C, Inoue H. Highly efficient gene replacements in Neurospora strains deficient for nonhomologous end-joining. Proc Natl Acad Sci U S A 2004; 101:12248-53.

107 Shinohara A, Ogawa H, Ogawa T. Rad51 protein involved in repair and recombination in $S$. cerevisiae is a RecA-like protein. Cell 1992; 69:457-70.

108 Wendland J. PCR-based methods facilitate targeted gene manipulations and cloning procedures. Curr Genet 2003; 44:115-23.

109McPherson MJ. PCR.Oxford: BIOS Scientific Publishers, 2000.

110Zarrin M, Leeder AC, Turner G. A rapid method for promoter 
exchange in Aspergillus nidulans using recombinant PCR. Fungal Genet Biol 2005; 42:1-8.

111 Davidson RC, Blankenship JR, Kraus PR, et al. A PCR-based strategy to generate integrative targeting alleles with large regions of homology. Microbiology 2002; 148:2607-15.

112 Yu JH, Hamari Z, Han KH, et al. Double-joint PCR: a PCR-based molecular tool for gene manipulations in filamentous fungi. Fungal Genet Biol 2004; 41:973-81.

113 Kamper J. A PCR-based system for highly efficient generation of gene replacement mutants in Ustilago maydis. Mol Gen Genomics 2004; 271:103-10.

114 Hamer L, Adachi K, Montenegro-Chamorro MV, et al. Gene discovery and gene function assignment in filamentous fungi. Proc Natl Acad Sci U S A 2001; 98:5110-5.

115 Adachi K, Nelson GH, Peoples, K.A, et al. Sequence analysis and functional characterization of the dialkylglycine decarboxylase gene DGD1 from Mycosphaearella graminicola. Curr Genet 2003; 43:358-63.

116 Takken FL, Van Wijk R, Michielse CB, et al. A one-step method to convert vectors into binary vectors suited for Agrobacteriummediated transformation. Curr Genet 2004; 45:242-8.

117 Jadoum J, Shadkchan Y, Osherov N. Disruption of the Aspergillus fumigatus $\arg B$ gene using a novel in vitro transposon-based mutagenesis approach. Curr Genet 2004; 45:235-41.

118Donofrio N, Rajagopalon R, Brown D, et al. PACLIMS: A component LIM system for high-throughput functional genomic analysis. BMC Bioinformatics 2005; 6:94.

119Arenz C, Schepers U. RNA interference: from an ancient mechanism to a state of the art therapeutic application? Naturwissenschaften 2003; 90:345-359.

120Forrest EC, Cogoni C, Macino G. The RNA-dependent RNA polymerase, QDE-1, is a rate-limiting factor in post-transcriptional gene silencing in Neurospora crassa. Nucleic Acids Res 2004; 32:2123-8.

121 Bernstein E, Caudy AC, Hammond SM, Hannon GJ. Role for a bidendate ribonuclease in the initiation step of RNA interference. Nature 2001; 409:363-6.

122 Catalanotto C, Azzalin G, Macino G, Cogoni C. Involvement of small RNAs and role of the qde genes in the gene silencing pathway in Neurospora. Genes Dev 2002; 16:790-5.

123 Kadotani N, Nakayashiki H, Tosa Y, Mayama S. RNA silencing in the phytopathogenic fungus Magnaporthe oryzae. Mol Plant Microbe Interact 2003; 16:769-76.

124Spiering MJ, Moon CD, Wilkinson HH, Schardl CL. Gene Clusters for insecticidal loline alkaloids in the grass-endophytic fungus Neotyphodium uncinatum. Genetics 2005; 169:140314.

125Goldoni M, Azzalin G, Macino G, Cogoni C. Efficient gene silencing by expression of double stranded RNA in Neurospora crassa. Fungal Genet Biol 2004; 41:1016-24.

126Mouyna I, Henry C, Doering TL, Latge JP. Gene silencing with RNA interference in the human pathogenic fungus Aspergillus fumigatus. FEMS Microbiol Lett 2004; 237:317-24.

127Nakayashiki H, Hanada S, Quoc NB, et al. RNA silencing as a tool for exploring gene function in ascomycete fungi. Fungal Genet Biol 2005; 42:275-83.

128 Gonzalez-Santos JM, Cao H, Wang A, et al. A complementation method for functional analysis of mammalian genes. Nucleic Acids Res 2005; 33:e94.

129Kars I, Krooshof GH, Wagemakers L, et al. Necrotizing activity of five Botrytis cinerea endopolygalacturonases produced in Pichia pastoris. Plant J 2005; 43:213-25.

130Luderer R, Takken FLW, de Wit PJGM, Joosten MHAJ. Cladosporium fulvum overcomes Cf-2-mediated resistance by producing truncated AVR2 elicitor proteins. Mol Microbiol 2002; 45:875-84.

131 Hamamoto H, Hasegawa K, Nakaune R, et al. Tandem repeat of a transcriptional enhancer upstream of the sterol 14a-demethylase gene (CYP51) in Penicillium digitatum. Appl Environ Microbiol 2000; 66:3421-6.

132Liu J, Sun SY, Wang TH. Construction of a yeast one-hybrid system with the xylanase 2 promoter from Trichoderma reesei to isolate transcriptional activators. Lett Appl Microbiol 2004; 38:277-82.

133 Saloheimo A, Aro N, Ilmen M, Penttila M. Isolation of the ace1 gene encoding a Cys(2)-His(2) transcription factor involved in regulation of activity of the cellulase promoter $c b h 1$ of Trichoderma reesei. J Biol Chem 2000; 275:5817-25.

134Duyvesteijn RGE, van Wijk R, Boer Y, et al. frpl is a Fusarium oxysporum $\mathrm{F}$-box protein required for pathogenicity on tomato. Mol Microbiol 2005; 57:1051-63.

135 Idurm A, Heitman J. Light controls growth and development via a conserved pathway in the fungal kingdom. PLOS Biol 2005; 3:615-26.

136Juvvadi P, Kuroki Y, Arioka M, Nakajima H, Kitamoto K. Functional analysis of the calcineurin-encoding gene cnaA from Aspergillus oryzae: evidence for its putative role in stress adaptation. Arch Microbiol 2003; 179:416-22.

137Hisada H, Hata Y, Kawato A, Abe, Y, Akita O. Cloning and expression analysis of two catalase genes from Aspergillus oryzae. J Biosci Bioeng 2005; 99:562-8.

138Bruno KS, Tenjo F, Li L, Hamer JE, Xu JR. Cellular localisation and role of kinase activity of PMK1 in Magnaporthe grisea. Eukaryot Cell 2004; 3:1525-32.

139Bachewich C, Masker K, Osmani S. The polo-like kinase PLKA is required for initiation and progression through mitosis in the filamentous fungus Aspergillus nidulans. Mol Microbiol 2005; 55:572-87.

140Bertossa RC, Kues U, Aebi M, Kunzler M. Promoter analysis of $\operatorname{cgl}$, a galectin encoding gene transcribed during fruiting body formation in Coprinopsis cinerea. Fungal Genet Biol 2004; 41:1120-31.

141 Howson R, Huh WK, Ghaemmaghami S, et al. Construction, verification and experimental use of two epitope-tagged collections of budding yeast strains. Comp Funct Genom 2005; 6:2-16.

142 Yang L, Ukil L, Osmani A, et al. Rapid production of gene replacement constructs and generation of a green fluorescent protein-tagged centromeric marker in Aspergillus nidulans. Eukaryot Cell 2004; 3:1359-62.

143 Schultz C, Schleifenbaum A, Goedhart J, Gadella jr TWJ. Multiparameter imaging for the analysis of intracellular signaling. Chembiochem 2005; 6:1323-30.

Edited by Lu Liang 